\title{
Brain Parenchymal Signal Abnormalities Associated with Developmental Venous Anomalies in Children and Young Adults
}

\author{
L.L. Linscott, J.L. Leach, B. Zhang, and B.V. Jones
}

\begin{abstract}
BACKGROUND AND PURPOSE: Abnormal signal in the drainage territory of developmental venous anomalies has been well described in adults but has been incompletely investigated in children. This study was performed to evaluate the prevalence of brain parenchymal abnormalities subjacent to developmental venous anomalies in children and young adults, correlating with subject age and developmental venous anomaly morphology and location.
\end{abstract}

MATERIALS AND METHODS: Two hundred eighty-five patients with developmental venous anomalies identified on brain MR imaging with contrast, performed from November 2008 through November 2012, composed the study group. Data were collected for the following explanatory variables: subject demographics, developmental venous anomaly location, morphology, and associated parenchymal abnormalities. Associations between these variables and the presence of parenchymal signal abnormalities (response variable) were then determined.

RESULTS: Of the 285 subjects identified, 172 met inclusion criteria, and among these subjects, 193 developmental venous anomalies were identified. Twenty-six (13.5\%) of the 193 developmental venous anomalies had associated signal-intensity abnormalities in their drainage territory. After excluding developmental venous anomalies with coexisting cavernous malformations, we obtained an adjusted prevalence of $21 / 181$ (11.6\%) for associated signal-intensity abnormalities in developmental venous anomalies. Signal-intensity abnormalities were independently associated with younger subject age, cavernous malformations, parenchymal atrophy, and deep venous drainage of developmental venous anomalies.

CONCLUSIONS: Signal-intensity abnormalities detectable by standard clinical MR images were identified in $11.6 \%$ of consecutively identified developmental venous anomalies. Signal abnormalities are more common in developmental venous anomalies with deep venous drainage, associated cavernous malformation and parenchymal atrophy, and younger subject age. The pathophysiology of these signal-intensity abnormalities remains unclear but may represent effects of delayed myelination and/or alterations in venous flow within the developmental venous anomaly drainage territory.

ABBREVIATIONS: $C M=$ cavernous malformation; $D V A=$ developmental venous anomaly; $P^{\prime}=a \chi^{2}$ test or Fisher exact test for categoric variables and the $t$ test or the Wilcoxon rank sum test for continuous variables; $P^{\prime \prime}=$ multivariate logistic regression models

$D$ evelopmental venous anomalies (DVAs) are frequently identified on routine MR imaging of the brain with contrast. DVAs are typically considered normal variants of venous development and usually have no associated imaging findings.

Received December 2, 2013; accepted after revision January 10, 2014.

From the Departments of Radiology (L.L.L., J.L.L., B.V.J.) and Biostatistics and Epidemiology (B.Z.); Cincinnati Children's Hospital Medical Center, University of Cincinnati College of Medicine, Cincinnati, Ohio.

Paper previously presented as an original research oral presentation at: Annual Meeting of the American Society of Neuroradiology, May 18-12, 2013; San Diego, California.

Please address correspondence to Luke Linscott, MD, Cincinnati Children's Hospital Medical Center, 3333 Burnet Ave, ML 5031, Cincinnati, OH 45229; e-mail: luke. linscott@cchmc.org

三 Indicates article with supplemental on-line tables

http://dx.doi.org/10.3174/ajnr.A3960
However, a subset of DVAs has been associated with findings such as cavernous malformations $(\mathrm{CMs}),{ }^{1-3}$ thrombosis with subsequent venous infarction, ${ }^{4-8}$ lobar atrophy, ${ }^{9}$ T2 and FLAIR signal-intensity abnormalities, ${ }^{9,10}$ and SWI hypointensities. ${ }^{11}$ Signal abnormalities can occur in the drainage territory of DVAs and may produce diagnostic uncertainty with regard to the significance and relationship to presenting symptoms. Signal abnormalities on MR imaging have been described in $12.5 \%{ }^{10}$ to $28.3 \%{ }^{9}$ of DVAs in adults, with an increasing prevalence with older age. While well described in adults, this relationship has not been investigated in children, to our knowledge. The MR imaging appearance of the brain in children is quite different from that in adults during myelination, and the effect of DVAs on regional brain maturation has not been studied. 
The most commonly proposed etiologies for parenchymal abnormalities associated with DVAs are chronic venous hypertension/insufficiency leading to ischemia or microhemorrhage. 9-12 $^{9}$ Although the effect of brain maturation is unknown, on the basis of these pathophysiologic mechanisms, we hypothesized that parenchymal abnormalities would be less common in children compared with adults. This study was performed to test this hypothesis and to investigate clinical factors and DVA characteristics associated with parenchymal signal abnormalities in children and young adults.

\section{MATERIALS AND METHODS Cohort Identification}

This retrospective study was approved by our institutional review board. The study was performed at a tertiary care children's hospital. A cutoff age of 35 years was chosen to ensure some age overlap with a similar study performed in adults. ${ }^{10}$ Using a radiology data base search engine (Softek Illuminate; Softek Solutions, Prairie Village, Kansas), we searched brain MR imaging with contrast reports for the term "developmental venous anomaly" from November 2008 to November 2012. This search resulted in the identification of 285 consecutive patients with developmental venous anomalies identified on brain MR imaging with contrast. The examinations were reviewed by a neuroradiologist (J.L.L.) and pediatric neuroradiology fellow (L.L.L.).

Criteria for inclusion in the study were the following: 1) subjects were younger than 35 years in age, 2) subjects had undergone MR imaging of the brain with contrast, and 3) the term "developmental venous anomaly" was used by the interpreting radiologist in the subject's final diagnostic radiology report. Exclusion criteria based on imaging included the following: incomplete/nondiagnostic examinations (defined as those examinations that did not include FLAIR or T2-weighted imaging or examinations limited by motion or other artifacts, 5 subjects), examinations in which the presence of a DVA was questionable on imaging review (23 subjects), and examinations in which the DVA was too small to characterize its morphology (35 subjects). Also excluded were subjects with underlying diagnoses that alone could explain the presence of parenchymal signal abnormalities, including tuberous sclerosis, neurofibromatosis type 1, intracranial vascular malformations (eg, Sturge-Weber Syndrome, dural arteriovenous fistulas, and so forth), and subjects who had undergone surgery involving brain parenchyma in the drainage territory of the DVA (50 subjects).

\section{Imaging}

One hundred thirty-six DVAs were scanned at 1.5T, and 57 DVAs were scanned at 3T. Typical 3T protocol included the following: a $3 \mathrm{D}$ volumetric T1-weighted gradient-echo sequence (TR, $10 \mathrm{~ms}$; TE, $4.6 \mathrm{~ms}$ ), an axial T2-weighted sequence (TR, $3000 \mathrm{~ms}$; TE, 100 $\mathrm{ms}$ ), and an axial T2-weighted FLAIR sequence (TR, 11,000 ms; TE, $125 \mathrm{~ms}$; TI, $2800 \mathrm{~ms}$ ). The $1.5 \mathrm{~T}$ protocol included the following: a sagittal T1-weighted FLAIR sequence (TR, $2200 \mathrm{~ms}$; TE, 26 $\mathrm{ms}$ ), an axial T2-weighted sequence (TR, $5000 \mathrm{~ms}$; TE, $85 \mathrm{~ms}$ ), and a T2 FLAIR sequence (TR, 10,000 ms; TE, $120 \mathrm{~ms}$; TI, 2200 $\mathrm{ms})$. Section thickness was $3-5 \mathrm{~mm}$ with a 0 - to $1-\mathrm{mm}$ intervening gap. Postcontrast imaging included a 3D volumetric T1 gradient- echo sequence for $3 \mathrm{~T}$ examinations and a T1-weighted FLAIR sequence in 3 planes for $1.5 \mathrm{~T}$ examinations. Gadolinium was administered at a dose of $0.2 \mathrm{mmol} / \mathrm{kg}$ intravenously.

\section{Image Analysis}

The examinations were reviewed in detail by 2 experienced radiologists. The first radiologist (J.L.L.) has 20 years of experience in interpreting MR imaging examinations and holds a Certificate of Added Qualification in neuroradiology. The second radiologist (L.L.L.) has 6 years of experience in interpreting MR imaging examinations and is currently a clinical fellow in pediatric neuroradiology.

DVA location was divided into 3 groups: 1) lobar, 2) basal ganglia/thalamus, and 3) brain stem/cerebellum. DVA morphology was described by using a previously published system devised by Lee et al. ${ }^{13}$ The DVAs were classified by depth as juxtacortical, subcortical, or periventricular. "Juxtacortical" depth was defined as within the gray matter or at the gray-white junction. "Subcortical" depth was defined as below the juxtacortical region but not adjacent to the ventricular wall. "Periventricular" depth was defined as adjacent to the lateral, third, or fourth ventricle or within the center of the structure, such as the pons. The drainage direction of the terminal or draining vein to which the venous radicles join was classified as either a deep (toward the ventricle) draining vein, superficial (toward the brain surface) draining vein, or both deep and superficial draining veins.

Images were specifically reviewed for the following: parenchymal atrophy, increased signal intensity on both FLAIR and T2weighted images, and cavernous malformations within the drainage territory of the DVA. "Cavernous malformations" were defined as focal lesions with T2 hypointense borders and associated exaggerated signal hypointensity on gradient recalled-echo or SWI sequences. Signal-intensity abnormalities associated with the DVA were defined as increased extravascular signal intensity on both FLAIR and T2-weighted images within the drainage territory of the DVA. ${ }^{9,10}$ The drainage territory was defined as the brain parenchyma directly adjacent to the visualized radicles of the DVA. Special care was taken to exclude increased signal intensity often seen within the venous radicles or draining vein, as described previously. ${ }^{10}$ In infants and young children, signal abnormality was defined as signal intensity qualitatively greater than that of surrounding white matter and white matter in the corresponding gyrus of the contralateral cerebral hemisphere.

The prevalence of signal-intensity abnormalities within the DVA drainage territory was correlated with the presence or absence of CM and parenchymal atrophy, as well as DVA location, depth of the draining vein, direction of the draining vein, age, and sex. For those patients with associated signal abnormality, follow-up duration and number of follow-up examinations were tabulated. Follow-up examinations were reviewed to determine whether the extent or character of parenchymal signal abnormality changed with time.

\section{Clinical and Imaging Findings Correlation}

Clinical indications and dominant imaging findings (other than the presence of a DVA) were identified from the radiology report (or electronic medical record, when necessary) for all DVAs with 

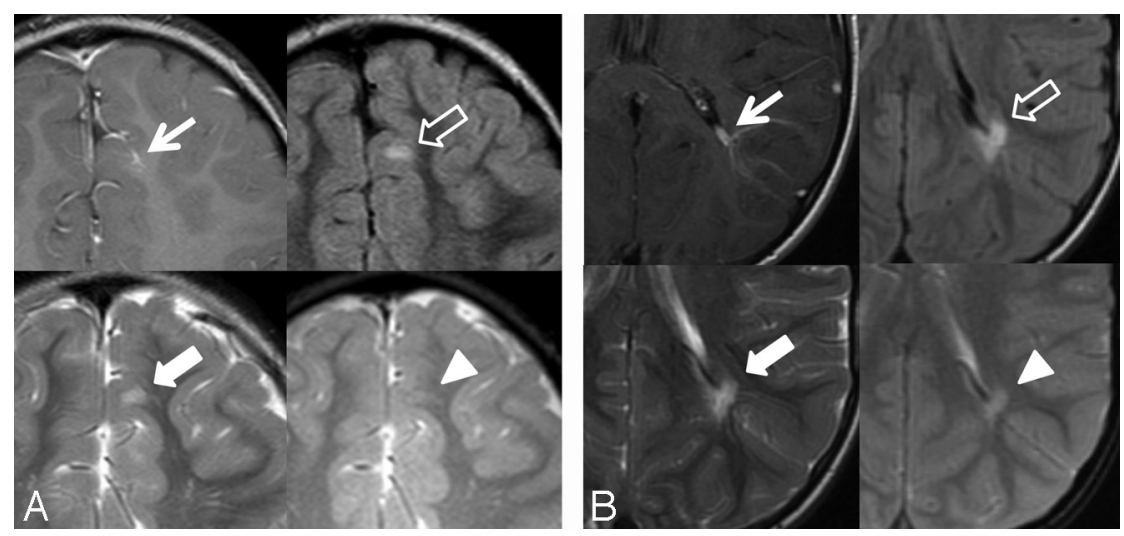

associated signal abnormalities were found in 2 patients with multiple DVAs. Cavernous malformations were identified in the drainage territory of 12 of the 193 (6.2\%) DVAs. Parenchymal atrophy was identified in 8 of the 193 (4.1\%) DVAs.

Twenty-six (13.5\%) of the 193 DVAs had associated signal-intensity abnormalities in their drainage territory. Five of the 26 DVAs with signal-intensity abnormalities were associated with cavernous malformations. After excluding the DVAs with associated CMs, an adjusted prevalence of 21/181 (11.6\%) for associated signal-intensity abnormalities in DVAs was obtained. No diffusion restriction was identified in the brain parenchyma adjacent to the signal abnormalities. Examples of DVA-associated signal abnormalities are shown in Fig 1.

and without associated signal abnormality. They were then correlated with the presence or absence of DVA-associated signal intensity in the DVA drainage territory.

\section{Statistical Analysis}

A $\chi^{2}$ test or Fisher exact test for categoric variables and the $t$ test or the Wilcoxon rank sum test for continuous variables $\left(P^{\prime}\right)$ were used to assess the relationship between signal change within the DVA drainage region (response variable) and DVA location, DVA depth, DVA drainage direction, age, sex, presence of atrophy or $\mathrm{CM}$, clinical indication, and additional MR imaging findings (explanatory variables). Values for the 2-sample $t$ test are expressed as mean $\pm \mathrm{SD}(95 \% \mathrm{CI})$ unless stated otherwise. OR for age was calculated as the likelihood of signal abnormality with each additional year. OR for sex was calculated as the likelihood of signal abnormality if the subject was female. ORs for focal atrophy and $\mathrm{CM}$ were calculated as the likelihood of signal abnormality in the presence of either associated abnormality. ORs for location, depth, and direction of the draining vein were calculated as the likelihood of signal abnormality compared with the first DVA morphologic descriptor (ie, lobar, periventricular, superficial), which was given an OR of 1.0. Multivariate logistic regression models $\left(P^{\prime \prime}\right)$ were used to evaluate the independent effects of age, sex, CM, parenchymal atrophy, location, depth, and direction of draining vein on signal change. Model selection under a stepwise criterion was performed to avoid multicollinearity. $P<.05$ was considered statistically significant. All analyses were performed by using SAS statistical software, Version 9.3 (SAS Institute, Cary, North Carolina.)

\section{RESULTS}

Of the 285 subjects initially identified in our data base search, 172 met the inclusion criteria. The mean age of the cohort was 10.8 years (14 months to 34 years) with an SD of 6.6 years and a 95\% CI of 9.9-11.8 years. The cohort consisted of 91 males and $81 \mathrm{fe}-$ males. In these 172 subjects, 193 DVAs were identified. Thirteen patients had $>1$ DVA (11 subjects had 2 DVAs identified and 2 subjects had $>2$ DVAs identified). Five of the 26 DVAs with
The Table outlines the association of signal abnormalities with other factors by using 193 DVAs from 172 subjects. The presence of increased FLAIR and T2 signal abnormality was associated with the presence of both CMs and parenchymal atrophy. Specifically, 5/26 (19.2\%) DVAs with signal abnormality were associated with CMs versus only $7 / 167$ (4.2\%) DVAs without signal abnormality $\left(P^{\prime}=.003, P^{\prime \prime}=.002\right)$. Five of $26(19.2 \%)$ DVAs with signal abnormality were associated with parenchymal atrophy versus $3 / 167(1.8 \%)$ DVAs without signal abnormality $\left(P^{\prime}<.001, P^{\prime \prime}=\right.$ $.004)$. Examples of CMs and parenchymal atrophy associated with signal-positive DVAs are shown in Figs 2 and 3, respectively.

There was a trend toward lobar location being associated with signal abnormality, but this did not reach statistical significance $\left(P^{\prime}=0.26, P^{\prime \prime}=0.589\right)$. The depth of the DVA was found to be associated with signal abnormality by using $\chi^{2}$ analysis, but it did not reach statistical significance by using a multivariate logistic regression analysis. Specifically, periventricular depth was more likely to be associated with signal abnormality than juxtacortical or subcortical depth. Eight of 27 (29.6\%) periventricular DVAs had associated signal abnormalities versus $8 / 96(8.3 \%)$ juxtacortical and 10/70 (14.3\%) subcortical DVAs $\left(P^{\prime}=.016, P^{\prime \prime}=.909\right)$. The direction of venous drainage was independently associated with signal abnormality. Specifically, deep or bidirectional venous drainage was more likely to be associated with signal abnormality than superficial venous drainage. Fourteen of 66 (21.2\%) deep and 3/6 (50\%) bidirectional draining DVAs had associated signal abnormalities versus $9 / 121(7.4 \%)$ superficial draining DVAs $\left(P^{\prime}=.001, P^{\prime \prime}=.047\right)$.

A lower subject age was associated with signal abnormality. Specifically, the median age of those subjects with signal abnormality was $7.3 \pm 5.5$ years (95\% CI, 5.1-9.5) compared with $11.4 \pm 6.6$ years $(95 \% \mathrm{CI}, 10.4-12.4)$ for those subjects without signal abnormalities $\left(P^{\prime}=.003, P^{\prime \prime}=.001\right)$. When subsets of age groups were further investigated, the prevalence of signal abnormalities was $5 / 19(26 \%)$ in subjects $12-36$ months, $12 / 68$ (17.6\%) in subjects $3-10$ years, $4 / 75$ (5.6\%) in subjects $11-20$ years, and $1 / 11(9.1 \%)$ in subjects older than 20 years of age (Fig 4). An 
Signal abnormalities related to subject age, sex, $\mathrm{CM}$, and parenchymal atrophy

\begin{tabular}{|c|c|c|c|c|c|c|}
\hline & $\begin{array}{c}\text { Signal } \\
\text { Abnormality }\end{array}$ & $\begin{array}{c}\text { No Signal } \\
\text { Abnormality }\end{array}$ & Total & $P^{\prime}$ & OR $(95 \% \mathrm{Cl})^{\mathrm{a}}$ & $P^{\prime \prime}$ \\
\hline $\begin{array}{l}\text { Total No. } \\
\text { Demographics }\end{array}$ & $26^{b}$ & $167^{c}$ & $193^{d}$ & & & \\
\hline Age & $7.3 \pm 5.5[5.1-9.5]$ & $11.4 \pm 6.6[10.4-12.4]$ & $10.8 \pm 6.6[9.9-11.8]$ & .003 & $0.84(0.76-0.94)$ & .001 \\
\hline Female & $10(38.5 \%)$ & $75(44.9 \%)$ & $85(44 \%)$ & .538 & $0.94(0.32-2.83)$ & .918 \\
\hline Associated abnormalities & & & & & & \\
\hline Focal atrophy & $5(17.8 \%)$ & $3(1.8 \%)$ & $8(4.1 \%)$ & $<.001$ & $17.1(2.52-117)$ & .004 \\
\hline CM & $5(19.2 \%)$ & $7(4.2 \%)$ & $12(6.2 \%)$ & .003 & $19.3(2.95-126)$ & .002 \\
\hline Location & & & & .260 & & .589 \\
\hline Lobar & $24(92.3 \%)$ & $129(77.2 \%)$ & $153(79.3 \%)$ & & 1.0 & \\
\hline Thalamus/BG & $0(0 \%)$ & $6(3.6 \%)$ & $6(3.1 \%)$ & & $0(0-\infty)$ & \\
\hline Cerebellum/BS & $2(7.7 \%)$ & 32 (19.2\%) & $34(17.6 \%)$ & & $0.37(0.05-2.49)$ & \\
\hline Depth of draining vein & & & & .016 & & .909 \\
\hline Periventricular & $8(30.8 \%)$ & $19(11.4 \%)$ & $27(14 \%)$ & & 1.0 & \\
\hline Subcortical & $10(38.5 \%)$ & $60(35.9 \%)$ & $70(36.3 \%)$ & & $0.73(0.18-3.01)$ & \\
\hline Juxtacortical & $8(30.8 \%)$ & $88(52.7 \%)$ & $96(49.7 \%)$ & & $0.81(0.16-4.09)$ & \\
\hline Direction of draining vein & & & & .001 & & .047 \\
\hline Superficial & $9(34.6 \%)$ & $112(67.1 \%)$ & $121(62.7 \%)$ & & 1.0 & \\
\hline Deep & $14(53.8 \%)$ & $52(31.1 \%)$ & $66(34.2 \%)$ & & $5.21(1.22-22.2)$ & \\
\hline Both & $3(11.6 \%)$ & $3(1.8 \%)$ & $6(3.1 \%)$ & & $6.85(0.84-56.0)$ & \\
\hline
\end{tabular}

Note:-BS indicates brain stem; BG, basal ganglia; $P^{\prime}$, univariate analysis ( $t$ test or $\chi^{2}$ test); $P^{\prime \prime}$, multivariate logistic regression; brackets, $95 \%$ confidence interval for age.

${ }^{a}$ OR for age is expressed as the likelihood of signal abnormality with each additional year. OR for sex is expressed as the likelihood of signal abnormality for a female subject. ORs for focal atrophy and CM are expressed as the likelihood of signal abnormality in the presence of either associated abnormality. ORs for location, depth, and direction of draining vein are expressed as the likelihood of signal abnormality compared with the first DVA morphologic descriptor (eg, lobar, periventricular, superficial), defined as a baseline OR of 1.0 .

$\mathrm{b} \%$ is the percentage of DVAs with a certain characteristic or associated finding divided by the total number of DVAs with signal abnormality.

$c \%$ is the percentage of DVAs with a certain characteristic or associated finding divided by the total number of DVAs without signal abnormality.

$d \%$ is the percentage of DVAs with a certain characteristic or associated finding divided by the total number of DVAs.
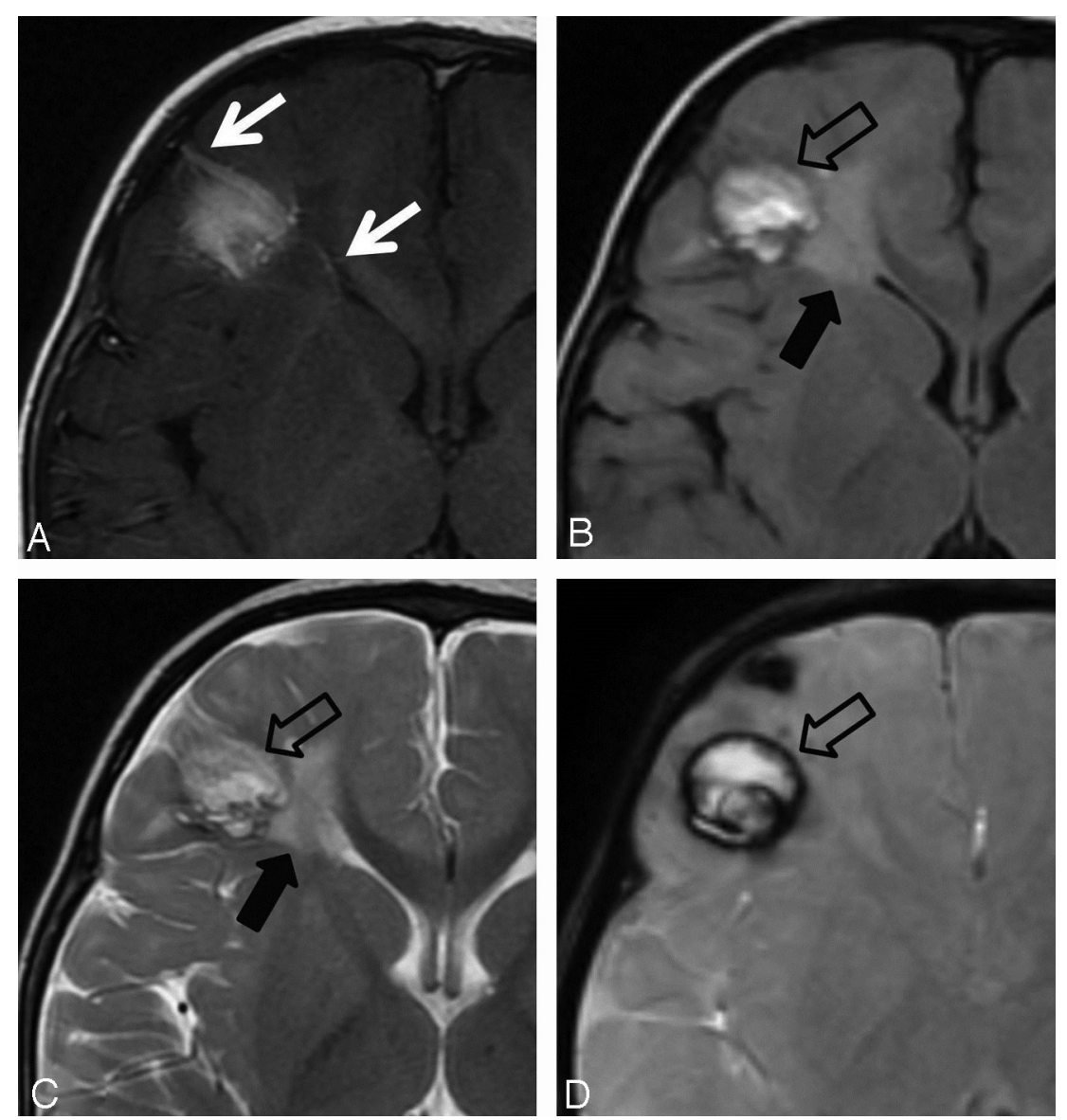

FIG 2. DVA with associated signal abnormality (black arrows) and CM (open black arrows) in a 13-month-old boy. A, TIWI with contrast. B, FLAIR. C, T2WI. D, SWI. Right frontal lobe DVA with subcortical depth and bidirectional venous drainage (white arrows). example of signal abnormality in the very young age group (12-36 months) is given in Fig 5. There was a trend toward more males with signal abnormalities than females, but this was not statistically significant $\left(P^{\prime}=\right.$ $\left..538, P^{\prime \prime}=.918\right)$. We found no association between clinical indication and signal abnormalities. Secondary imaging findings of CMs and atrophy remote from the DVA were associated with signal abnormalities (On-line Table 1). Secondary imaging findings of remote intracranial mass were associated with the absence of signal abnormality (On-line Table 2).

Twenty-one of 26 (80.1\%) DVAs with associated signal abnormality had follow-up examinations with a median follow-up of 26 months (1-42 months). The median number of follow-up MR imaging examinations for these 21 DVAs was 2 (1-7 examinations). There was no change in the extent or character of signal abnormalities on follow-up in 19 of 21 DVAs with associated signal abnormality alone. Two DVAs with signal abnormality alone showed a subtle decrease in signal abnormality on follow-up examinations. These patients were 2 years and 17 months of age at the time of initial study with a follow-up of 3 and 4 years, respectively (Fig 6). Two of 

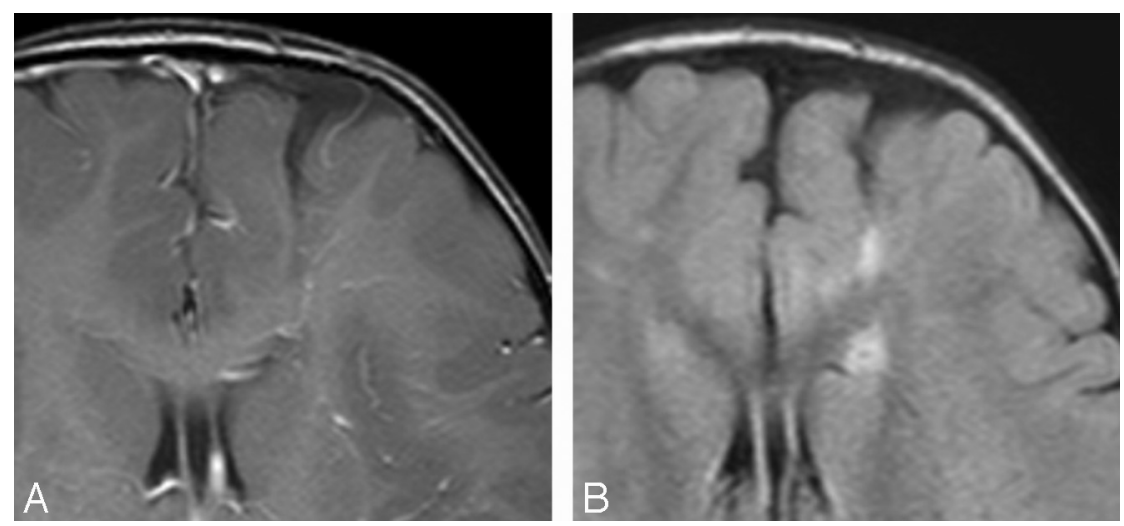

FIG 3. DVA in a 23-month-old boy with associated signal abnormality and parenchymal atrophy. A, TIWI with contrast. B, FLAIR. Left frontal lobe DVA with periventricular depth and deep venous drainage. Note the increased FLAIR and T2 signal abnormality with associated parenchymal atrophy.

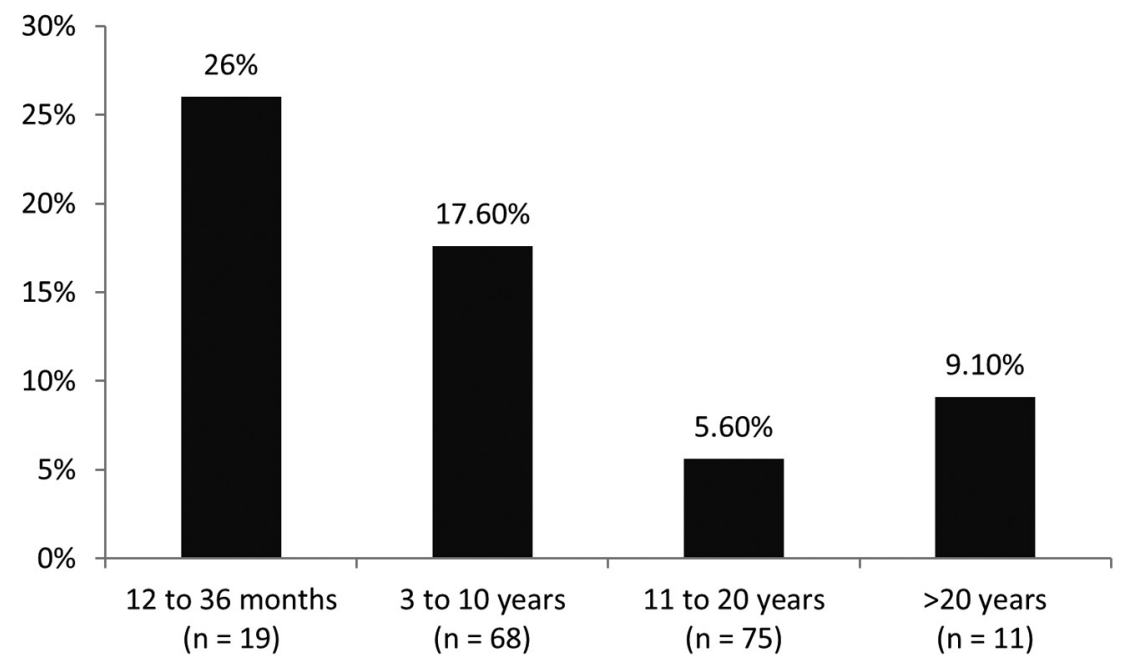

FIG 4. Percentage signal abnormalities associated with DVAs by age group. $n$ indicates the number of subjects in each age group.

the 5 DVAs with signal abnormality and associated CMs showed a decrease in signal hyperintensity on follow-up examinations.

\section{DISCUSSION}

The major finding of this study is that in children and young adults, signal-intensity abnormalities detectable by MR imaging were identified in $11.6 \%$ of consecutively identified DVAs. This is similar to the prevalence identified in prior investigations of adult patients. ${ }^{10}$ Although not proved, our results suggest that these signal abnormalities occur with a higher prevalence in younger patients and may decrease with brain maturation. To our knowledge, this is a novel finding because change in signal with time has not been identified in any of the similar studies performed in adults. ${ }^{9-11}$ Additional parenchymal abnormalities such as CMs and parenchymal atrophy were strongly associated with signal abnormalities. Finally, we identified deep venous drainage as the only morphologic characteristic of DVAs that is independently predictive of associated signal abnormality.

The etiology of parenchymal signal abnormalities associated with DVAs in adults and children is incompletely understood. Prior investigators have suggested that abnormal FLAIR signal intensity may represent edema or gliosis secondary to chronic venous insufficiency/ hypertension related to anomalous venous drainage. ${ }^{9,10}$ Altered hemodynamics in the drainage territory of DVAs has been established with case reports that described increased perfusion parameters in "atypical" DVAs. ${ }^{14,15}$ More recently, a larger study of DVAs found increased relative $\mathrm{CBV}$, relative $\mathrm{CBF}$, and $\mathrm{MTT}$ in most DVA studies. ${ }^{12}$ Additionally, they found that those DVAs with associated CMs had higher MTT values than those DVAs without CMs, suggesting that hemodynamic factors may influence parenchymal manifestations of DVAs. A similar study of perfusion parameters in the setting of white matter signal abnormalities would be informative. Perfusion was performed in too few of our cases to evaluate its possible contribution to signal abnormalities. Other studies have suggested that stenosis of the draining vein may cause venous hypertension/insufficiency. ${ }^{16,17}$ A recent investigation of morphologic factors associated with CMs and DVAs found tortuosity of the medullary veins and angulation of the draining vein to be associated with the presence of a CM in the DVA territory. ${ }^{18}$ This finding also supports the hypothesis that hemodynamic variables may impact the presence of parenchymal abnormalities. Given the small size of most DVAs in our study, resolution was not adequate to assess draining vein stenosis and medullary vein tortuosity.

Based on these prior investigations of parenchymal abnormalities associated with DVAs, our hypothesis was that the prevalence of white matter signal abnormalities would be less common in children than in adults. Contrary to our hypothesis, we found a nearly equal prevalence of brain parenchymal signal abnormalities in children and young adults (median age, 10 years) (11.6\%) compared with older adults (median age, 47 years) $(12.5 \%){ }^{10}$ Most interesting, when we performed a subset analysis by age group, we identified an unexpected inverse relationship between subject age and signal abnormality, with the highest prevalence being found in the youngest subjects and the lowest prevalence in teenagers. When we compared our findings with those of Santucci et al, ${ }^{10}$ which found a higher prevalence of signal abnormalities in older patients, a bimodal age distribution of signal abnormalities is suggested. The highest prevalence of signal abnormalities occurs in the very young, steadily decreases to a nadir in the teenage and early adult years, and then increases with age. One possible explanation for this finding is delayed myelination in the drainage territory of the DVA in young children. Perhaps alterations in the venous pressure within the DVA cause delayed egress of inter- 


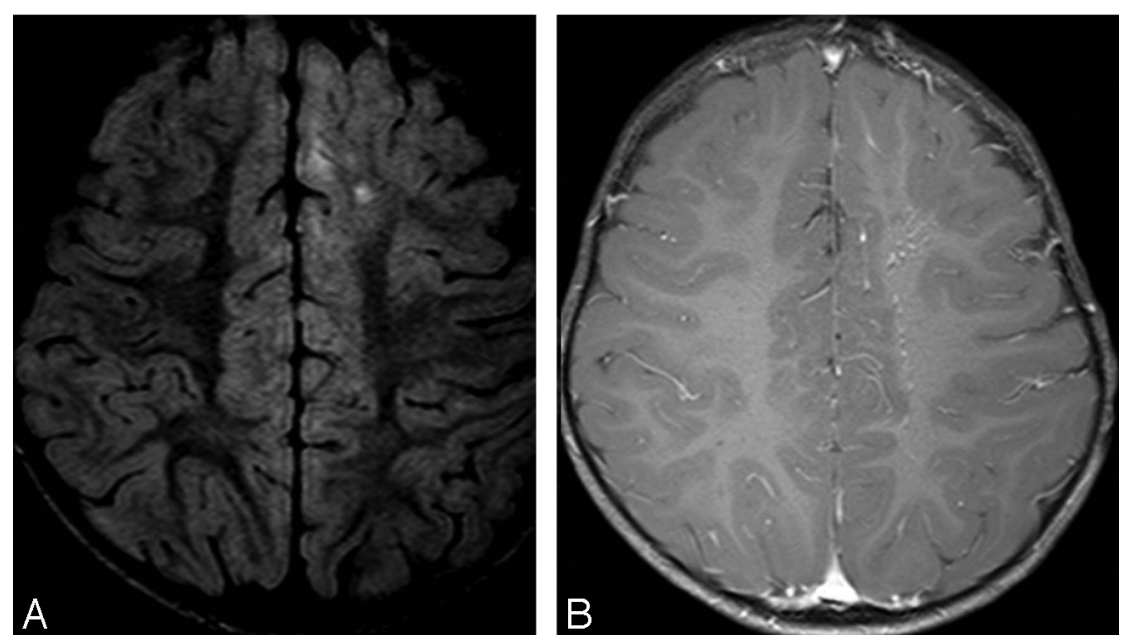

FIG 5. FLAIR $(A)$ and TTWI with contrast $(B)$ show signal abnormality in the images of a 23-monthold boy, showing relative increased signal within the drainage territory of the DVA compared with normal contralateral myelinating white matter. Left frontal lobe DVA with periventricular depth and deep venous drainage.
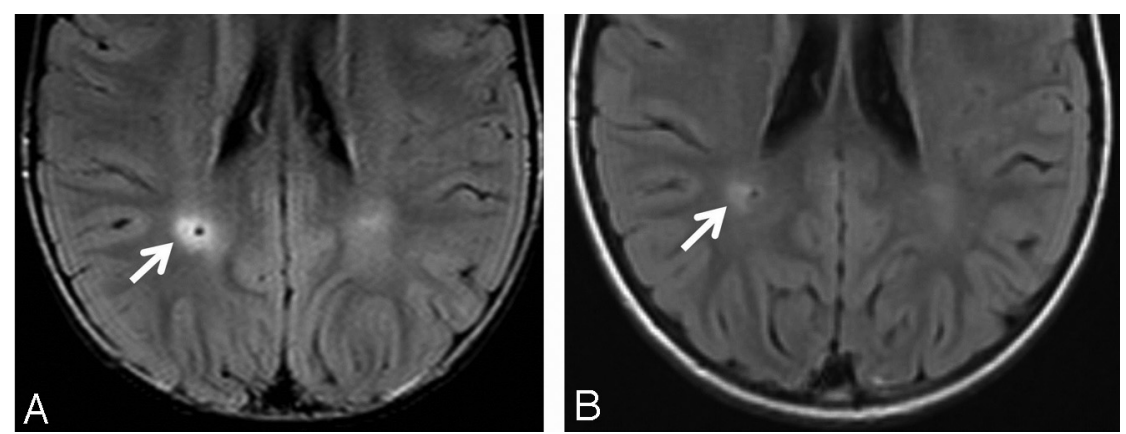

FIG 6. DVA with a subtle decreased extent of signal abnormality on follow-up examination. Axial FLAIR images at 2 years $(A)$ and 5 years $(B)$ of age. Note subtle decreased signal intensity in the drainage territory of the DVA along the lateral aspect of the DVA draining vein.

stitial water as myelination progresses in infancy and early childhood, leading to relatively increased signal intensity in the brain parenchyma drained by the DVA. ${ }^{19}$

If the higher prevalence of signal abnormalities in a younger population is to be explained by delayed myelination, we would expect some percentage of these signal abnormalities to decrease or resolve with time with progressive myelination. In fact, this was our observation, because 2 of the 21 DVAs with signal abnormality alone did demonstrate subtle decreased signal abnormality on follow-up examinations. On the basis of our study and the existing adult literature, it is interesting to speculate that myelination changes may be primarily responsible for signal abnormalities in the DVA drainage territory in infants and young children, resolving or becoming less apparent in older children and adolescents. With aging, gliosis may become a more dominant process, becoming nonreversible on imaging evaluation. Additional investigations with larger populations of young children, expanded mixed-age populations, and more subjects with long-term follow-up will be needed to confirm these age associations.

As we investigated the possible association of DVA morphology and location with the presence of signal abnormality, we identified deep venous drainage as the only independently predictive variable. The physiologic basis for this association is uncertain, but perhaps cerebral deep venous drainage has less venous flow capacity and may be more likely to cause venous insufficiency/hypertension. Deep venous drainage has been identified as a significant predictor of hemorrhage in arteriovenous malformations. ${ }^{20-22}$ We found no significant association between the location of the DVA and signal abnormality. This finding is supported by a similar study in adults, which found no association with DVA location. ${ }^{10}$ The DVA depth was associated with signal abnormality when using univariate analysis, but not when using a multivariate analysis, suggesting that the influence of depth on signal is related, in some fashion, to the direction of drainage.

In the course of our investigation of white matter signal abnormalities, we were interested in identifying the prevalence of other well-documented parenchymal abnormalities associated with DVAs, namely $\mathrm{CMs}^{1-3}$ and parenchymal atrophy. ${ }^{9}$ The prevalence of CMs (6.2\%) in our study population is similar to that of 2 previously reported studies investigating the association of DVAs and CMs in adults, which found CMs in $3.4 \%{ }^{10}$ and $13.3 \%$ of DVAs. ${ }^{9}$ This outcome was contrary to our expectations because many have suggested that the development of CMs in the DVA drainage territory is a dynamic process that evolves with time, beginning with small microhemorrhages within the drainage territory of DVAs. ${ }^{23-27}$ Under such a scenario, one would expect the prevalence of CMs associated with DVAs to increase with time and be more common in adults than in children. We also found that those DVAs with CMs were more likely to have associated signal abnormality. Despite the small number of cavernous malformations, this association reached statistical significance. The association between signal abnormality and CMs is problematic because the etiology of the signal abnormality may not be confidently attributed to the presence of the DVA alone. One could argue that the signal abnormality is secondary to hemorrhage of the $\mathrm{CM}$; however, this association between signal abnormality and cavernous malformations is supported by a recent investigation of DVAs in adults by using susceptibility-weighted imaging to identify the prevalence of hypointense foci on SWI within the drainage territory of DVAs. ${ }^{11}$ The study found that white matter hyperintense lesions were more frequently observed in patients with hypointense SWI foci versus those without hypointense SWI foci. Additionally, the study found a $62 \%$ prevalence of SWI hypointense foci in the study population, which is much higher than that in our study. This discrepancy may, in part, be explained by the relatively small number of examinations that included SWI in our study. Alternatively, SWI hypointense foci may be less com- 
monly encountered in the pediatric population. A dedicated examination of hypointense foci in children by using SWI is warranted.

In regard to parenchymal atrophy and associated signal abnormalities, we found a low prevalence of parenchymal atrophy (4.1\%), significantly less than that in a study of adults $(29.7 \%){ }^{9}$ This may be related to the development of localized atrophy with increasing age but could also be related to selection bias used in some prior investigations (ie, potentially selecting only larger DVAs for evaluation). Atrophy was more commonly encountered in those DVAs with associated signal abnormality, suggesting that these 2 parenchymal abnormalities may share common etiologic factors such as venous hypertension/insufficiency.

We found no association between clinical indication and signal abnormalities, suggesting that these signal abnormalities are predominantly asymptomatic or have no typical presenting symptoms associated with them. When we evaluated possible associations with secondary imaging findings and signal abnormalities, we found an association between CMs and atrophy remote from the DVA. The association of atrophy remote from the DVA is noteworthy. It may be that factors contributing to remote atrophy (eg, radiation, ischemia, systemic venous hypertension, and so forth) make the development of signal abnormality in the DVA territory more likely, especially if the altered hemodynamics within the DVA territory predispose this brain parenchyma to such injury. Finally, a peculiar association identified in our study was that DVAs without signal abnormality were associated with a secondary imaging finding of a remote intracranial mass. We have no explanation for this association, and it may represent a statistical anomaly.

Our study does have some limitations. Case selection was based initially on imaging reports. Some small DVAs may have been missed and therefore not selected for our study cohort. Clinical indications were often obtained from the study indication and may not fully represent the patient's clinical presentation. The relatively small number of subjects limits our ability to compare DVA characteristics and signal change between age subgroups. The differentiation between DVA-related parenchymal signal changes and intrinsic DVA-related signal can be problematic; however, it has been applied successfully in prior studies by our group. ${ }^{10}$

\section{CONCLUSIONS}

Signal-intensity abnormalities detectable by standard clinical MR images were identified in 13.5\% of consecutively identified DVAs in children and young adults $(11.6 \%$ adjusted prevalence when CMs were excluded). Signal abnormalities were more common in DVAs with associated CM and parenchymal atrophy, with deep venous drainage, and in younger subjects in our cohort. The pathophysiology of these signal-intensity abnormalities remains unclear but may represent effects of delayed myelination and/or alterations in venous flow within the DVA drainage territory. More work in this area is warranted.

Disclosures: James L. Leach—UNRELATED: Grants/Grants Pending: Eunice Kennedy Shriver National Institute of Child Health and Human Development (HHSN275200900018C Pediatric Functional Imaging Research Network).* *Money paid to the institution.

\section{REFERENCES}

1. Abe T, Singer RJ, Marks MP, et al. Coexistence of occult vascular malformations and developmental venous anomalies in the central nervous system: MR evaluation. AJNR Am J Neuroradiol 1998; 19:51-57

2. Beall DP, Bell JP, Webb JR, et al. Developmental venous anomalies and cavernous angiomas: a review of the concurrence, imaging, and treatment of these vascular malformations. J Okla State Med Assoc 2005;98:535-38

3. Perrini P, Lanzino G. The association of venous developmental anomalies and cavernous malformations: pathophysiological, diagnostic, and surgical considerations. Neurosurg Focus 2006; 21:e5

4. Ballarin R, Di Benedetto F, De Ruvo N, et al. Thrombosis of developmental venous anomalies of the brain after liver transplantation. Transplantation 2009;87:615-16

5. Dorn F, Brinker G, Blau T, et al. Spontaneous thrombosis of a DVA with subsequent intracranial hemorrhage. Clin Neuroradiol 2013;23:315-57

6. Kiroglu Y, Oran I, Dalbasti T, et al. Thrombosis of a drainage vein in developmental venous anomaly (DVA) leading venous infarction: a case report and review of the literature. I Neuroimaging 2011;21:197-201

7. Lai PH, Chen PC, Pan HB, et al. Venous infarction from a venous angioma occurring after thrombosis of a drainage vein. AJR Am J Roentgenol 1999;172:1698-99

8. Teo M, St George J, Jenkins S, et al. Developmental venous anomalies: two cases with venous thrombosis. Br J Neurosurg 2012;26:886-87

9. San Millán Ruíz D, Delavelle J, Yilmaz H, et al. Parenchymal abnormalities associated with developmental venous anomalies. Neuroradiology 2007;49:987-95

10. Santucci GM, Leach JL, Ying J, et al. Brain parenchymal signal abnormalities associated with developmental venous anomalies: detailed MR imaging assessment. AJNR Am J Neuroradiol 2008;29:1317-23

11. Takasugi M, Fujii S, Shinohara Y, et al. Parenchymal hypointense foci associated with developmental venous anomalies: evaluation by phase-sensitive MR imaging at 3T. AJNR Am J Neuroradiol 2013;34:1940-44

12. Sharma A, Zipfel GJ, Hildebolt C, et al. Hemodynamic effects of developmental venous anomalies with and without cavernous malformations. AJNR Am J Neuroradiol 2013;34:1746-51

13. Lee C, Pennington MA, Kenney CM, 3rd. MR evaluation of developmental venous anomalies: medullary venous anatomy of venous angiomas. AJNR Am J Neuroradiol 1996;17:61-70

14. Kroll H, Soares BP, Saloner D, et al. Perfusion-CT of developmental venous anomalies: typical and atypical hemodynamic patterns. J Neuroradiol 2010;37:239-42

15. Camacho DL, Smith JK, Grimme JD, et al. Atypical MR imaging perfusion in developmental venous anomalies. AJNR Am J Neuroradiol 2004;25:1549-52

16. Truwit CL. Venous angioma of the brain: history, significance, and imaging findings. AJR Am J Roentgenology 1992;159:1299-307

17. Dillon WP. Cryptic vascular malformations: controversies in terminology, diagnosis, pathophysiology, and treatment. AJNR Am J Neuroradiol 1997;18:1839-46

18. Hong YJ, Chung TS, Suh SH, et al. The angioarchitectural factors of the cerebral developmental venous anomaly: can they be the causes of concurrent sporadic cavernous malformation? Neuroradiology 2010;52:883-91

19. Branson HM. Normal myelination: a practical pictorial review. Neuroimaging Clin N Am 2013;23:183-95

20. Kader A, Young WL, Pile-Spellman J, et al. The influence of hemodynamic and anatomic factors on hemorrhage from cerebral arteriovenous malformations. Neurosurgery 1994;34:801-07, discussion 807-08 
21. Langer DJ, Lasner TM, Hurst RW, et al. Hypertension, small size, and deep venous drainage are associated with risk of hemorrhagic presentation of cerebral arteriovenous malformations. Neurosurgery 1998;42:481-86, discussion 487-89

22. Turjman F, Massoud TF, Vinuela F, et al. Correlation of the angioarchitectural features of cerebral arteriovenous malformations with clinical presentation of hemorrhage. Neurosurgery 1995;37: 856-60, discussion 860-62

23. Maeder P, Gudinchet F, Meuli R, et al. Development of a cavernous malformation of the brain. AJNR Am J Neuroradiol 1998;19:1141-43

24. Cakirer S. De novo formation of a cavernous malformation of the brain in the presence of a developmental venous anomaly. Clin Radiol 2003;58:251-56

25. Campeau NG, Lane JI. De novo development of a lesion with the appearance of a cavernous malformation adjacent to an existing developmental venous anomaly. AJNR Am J Neuroradiol 2005; 26:156-59

26. Awad IA, Robinson JR, Jr, Mohanty S, et al. Mixed vascular malformations of the brain: clinical and pathogenetic considerations. Neurosurgery 1993;33:179-88, discussion 188

27. Rigamonti D, Spetzler RF, Medina M, et al. Cerebral venous malformations. J Neurosurg 1990;73:560-64 